\title{
Ist Konsens Nonsens?
}

$\mathrm{K}$ onsens ist Nonsens könnte man meinen, wenn man in den letzten Wochen Berufsverband (BDU) und Fachgesellschaft (DGU) beobachtet. Bekanntlich haben beide Vereine unterschiedliche Aufgaben: Der Berufsverband ist die Interessenvertretung der niedergelassenen Urologen als Unternehmer. Wichtiges Ziel ist es, den Rahmen der Berufsausübung für seine Mitglieder sinnvoll zu gestalten. Er fördert die Pflege des Gemeinschaftsgeistes unter den Urologen und unterhält gute Kontakte zur DGU - wahr(schein)lich eine schwierige Aufgabe. Da hat es die wissenschaftliche Fachgesellschaft leichter; sie ist die Interessenvertretung der Ärzte als Wissenschaftler und sollte Wissenschaft, Lehre, Fort- und Weiterbildung sowie Krankenversorgung fördern. Die Gefahr der Dualität ist gegeben: hier die Niedergelassenen, dort die Kliniker mit unterschiedlicher Interessenlage. Umso wichtiger ist das Motto der Einheitsfront: getrennt marschieren, vereint schlagen!

Stattdessen ist der Dissens allerorten spürbar, die Diskussion vergiftet und es gibt nur Verlierer. Im Zusammenspiel der beiden Organisationen geht es leider immer wieder um Personen und künstliche Abgrenzungen in den Gremien und weniger um Inhalte. Die erfordern aber viel Gemeinsamkeit. Zum Beispiel müssen unsere niedergelassenen Kollegen wissenschaftlich zur Teilnahme an der klinischen Forschung und der Versorgungsforschung motiviert und dabei betreut werden. Das ist eine Aufgabe, bei der BDU als Mittler und DGU als Hüterin der Wissenschaft Hand in Hand arbeiten müssten mit dem Ziel: Erkenntnisse durch Daten. Mit dem neuen Arbeitskreis „Versorgungsforschung“ der Fachgesellschaft ist das nicht getan, denn die meisten der 21 Arbeitskreise haben nur eine Alibifunktion und bedürften der Reorganisation, um die Aktivisten von den „Schläfern“ zu trennen. Einige der über 377 Mitglieder „arbeiten " in den Führungsgremien - das sind vorwiegend Kliniker. Ihre Heilkunst beschränkt sich zumeist auf Anwendung verschiedener Operationstechniken. Sie erleben ihre Patienten nur während des kurzen stationären Aufenthaltes, betrachten also die Versorgungskette durch eine Spaltlampe und kennen nur Fragmente ihrer eigenen Ergebnisse. Nach der Rückkehr in die ambulante Versorgungswelt kann wieder durch das Weitwinkelobjektiv beobachtet werden, das heißt, die Niedergelassenen kennen die Langzeitverläufe und deshalb wären sie Herren über die Daten mit den Ergebnissen aus dem Versorgungsgeschehen. Dabei darf es ihnen an der wissenschaftlichen Anleitung und Unterstützung durch die Fachgesellschaft nicht fehlen, wenn es beispielsweise darum geht, Studien zu initiieren oder fortzuführen, die für das Wohl unserer Patienten wichtig sind und die vor allem die Versorgungsrealität abbilden. Die Vision des Berufsverbandes muss es sein, bei seinen Mitgliedern den Wunsch nach Erkenntnis zu wecken und zu realisieren. Mit der DGU sollte deshalb gemeinsam „sauberes Wissen“ generiert werden. Die Kooperationsarchitektur zwischen BDU und Fachgesellschaft wäre ein virtuelles dreigeschossiges „Haus des Wissens“. Hier würden im Erdgeschoss Programme zur Datenakquise im Rahmen der Versorgungsforschung entwickelt und durchgeführt (Wissensgenerierung). Die Belle Etage erteilte Aufträge an einschlägige Institutionen wie äzq oder Cochrane, um Forschungsergebnisse aus Literatur und Studien zu sichten und zu beurteilen (Wissensaufbereitung). Das Obergeschoss sorgte dann für deren Kommentierung und Verbreitung (Wissenstransfer). So wäre die Urologie endlich auf dem Wege zu einer wirklichen Wissensgesellschaft, ohne die Vielzahl teurer, redundanter, überflüssiger Kongresse und Tagungen, auf denen Literatur oft genug fehlinterpretiert wird.

Ärzte haben sich deprofessionalisieren lassen, indem sie in ihrem ehemaligen Standesberuf die Selbstständigkeit verloren haben (P. Unschuld „Ware Gesundheit - das Ende der klassischen Medizin“, C.H. Beck 2011). Sie können ihre Tätigkeit nicht mehr nach bewährten fachlichethischen Kriterien ausüben: In der Klinik stehen ihnen die „Propheten der Effizienz" entgegen und winken mit Dienstvertrag und jährlichen Zielvereinbarungen, in der Praxis sind es patientenferne Leistungsregulatoren und falsche Anreizsysteme. Die Chancen liegen in einer Allianz, um mit einer Stimme zu sprechen und um die ärztliche Kompetenz in einer komplexer werdenden Versorgungslandschaft wieder zu stärken.

Fazit: ohne Konsens ist alles Nonsens.

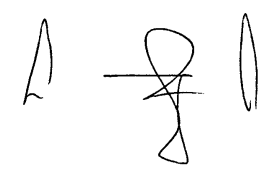

